\title{
PL-2: Vacuum Electronics Research Perspective at the Naval Research Laboratory
}

\author{
Gerald M. Borsuk and Baruch Levush \\ US Naval Research Laboratory, Washington, DC 20375 USA
}

\begin{abstract}
The US Naval Research Laboratory (NRL) is actively engaged in research and development of key technologies for vacuum electronics high-power amplifiers at frequencies ranging from $30 \mathrm{GHz}$ to $1000 \mathrm{GHz}$. This work includes the development and application of physics-based modeling and simulation tools, the design and development of emittance dominated pencil and spatially distributed electron beams and associated slowwave interaction structures, research on high-currentdensity emitters, the development and application of precise micro-fabrication techniques and thermal management schemes, and the development of metrology techniques in the $\mathrm{THz}$ regime. An overview of these activities will be presented, including a discussion of the technological advances required to achieve order-ofmagnitude increases in amplifier performance. A summary of the approaches being pursued and the status of these efforts will also be presented. Future research opportunities for vacuum electronics at NRL will also be discussed.
\end{abstract}

Keywords: Vacuum electronics; amplifier; millimeter wave; slow wave; THz; distributed beam; sheet beam.

\section{Background}

Coherent electromagnetic radiation with sufficient power in the millimeter-wave and $\mathrm{THz}$ spectrum (30 to 1000 $\mathrm{GHz}$ ) can be used in variety of very demanding systems applications: high-data-rate communication links, radars with exacting resolution capabilities in complex environments, high-resolution imaging, and biological and chemical detectors are examples. Many modern platforms are prime power and space limited. Such platforms require multifunctional systems with a substantial increase in specific power (power per unit volume and weight) and efficiency over existing capabilities. Unfortunately, it is difficult to implement these types of systems because there are currently no amplifiers available at these frequencies that exhibit the necessary power, efficiency, linearity, and instantaneous bandwidth simultaneously.

The technological options for generating high power in the millimeter-wave and $\mathrm{THz}$ spectrum have been limited to vacuum electronic devices based on fast-wave interactions (e.g., gyrotrons). They are capable of producing very high powers in this frequency range but at the expense of requiring superconducting or pulsed magnets. High power from compact slow-wave-devices has lagged because of the difficulty of generating and propagating high current density beams through small interaction circuits having dimensions on the order of a fraction of a wavelength (e.g. $\sim 500$ microns or less). Recently, interest in achieving high power coherent EM radiation in the millimeter-wave and $\mathrm{THz}$ spectrum using slow-wave devices has been revived [1] because of developments in micro-fabrication techniques combined with newly developed three dimensional simulation codes for modeling the electron beam formation, transport and beam-wave interaction [2].

Significant improvements in the performance of conventional pencil-beam vacuum electronics amplifiers have been made in this frequency regime over the past decade or so. However further improvements are restricted by fundamental physical limitations. To overcome these limitations, new approaches are required. Much of the work at the Naval Research Laboratory (NRL) has been focused on investigating approaches that enable generation and transport of higher current electron beams, particularly sheet beams and multiple beams that permit significantly higher current to be transported through a circuit at a given voltage than is possible with pencil beams (assuming the current density can be maintained). These distributed beam amplifiers offer the prospect of considerably higher power and specific power than can be achieved with comparable pencil beam devices.

\section{Distributed Beam Amplifiers}

The topological approach for distributed beam amplifiers is shown in Figure 1, where a quasi-sheet beam, consisting of many closely spaced pencil beams in a common interaction structure (a), is generalized to a sheet beam (b). These basic distributed beam units/modules can be combined in a co-planar array of the required number to achieve the desired total power. The concept of a coplanar array is motivated by the limitations to total current that can be achieved at reasonable, sustainable current densities in the presence of beam instabilities and mode competition, both of which limit quasi-sheet beam or sheet beam width.

A number of key obstacles must be overcome to achieve the full potential of spatially distributed-beam amplifiers. They include the following: 1) the creation of high brightness electron sources, i.e. high current density, low emittance and long life cathodes; 2) the formation and transport of a sufficiently high-perveance beam, 3 ) the design 
and fabrication of suitable interaction circuits, and 4) the suppression of parasitic modes.

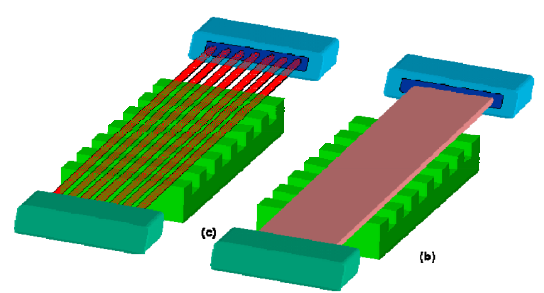

Figure 1. (a) Quasi-sheet beam, i.e., many closely spaced pencil beams with a common interaction structure, (b) sheet beam.

The figure of merit, $P f^{2}$, is a useful measure of coherent radiation power density of an amplifier, where $P$ is the average power and $f$ is the operating frequency. To measure the ability of the device to transmit information, the appropriate figure of merit is $P f^{2}(\Delta f / f)$, where $(\Delta f / f)$ is the fractional instantaneous bandwidth of an amplifier and is presented in Fig. 2 for frequencies above $20 \mathrm{GHz}$. Stateof-the-art capabilities of both solid state and vacuum electronics amplifiers are plotted together with the vacuum electronics amplifiers performance goals of the NRL research programs. NRL is also actively engaged in two DARPA programs, HiFIVE $(220 \mathrm{GHz}$ amplifiers $)$ and $\mathrm{THz}(670 \mathrm{GHz}, 850 \mathrm{GHz}$ and $1030 \mathrm{GHz}$ amplifiers). The $\mathrm{THz}$ program vacuum electronics amplifiers performance goals do not require a spatially distributed-beam. However the challenges listed above are very similar for the $\mathrm{THz}$ amplifiers as well.

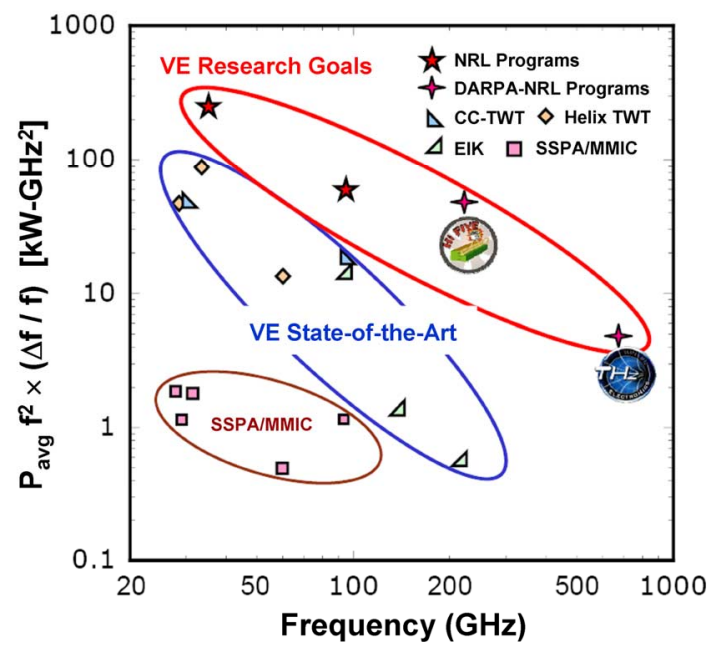

Figure 2. State-of-the-art and prospective devices described in terms of (Pavg $\mathrm{f} 2)(\Delta \mathrm{f} / \mathrm{f})$ figure of merit.

\section{Summary}

Progress in NRL conducted research activities addressing many of the key issues for achieving significant power, efficiency, linearity, and instantaneous bandwidth in the millimeter and sub-millimeter electromagnetic spectrum will be highlighted. Additional technical details, such as emission physics [3], novel cathodes [4], beam formation [5] and beam transport [6], micro-fabrication techniques [7], novel circuits [8]-[11], numerical analysis of nonlinear beam-wave interaction [12]-[13] and new design codes [14]-[17] are reported at this conference.

This work has been funded by the US Office of Naval Research.

\section{References}

[1] J. H. Booske, "Plasma physics and related challenges of millimeter-wave-to-terahertz and high power microwave generation," Physics of Plasmas, vol. 15, 2008.

[2] L. D. Ludeking, et al. in Modern Microwave and Millimeter-Wave Power Electronics, edited by R. J. Barker, J. H. Booske, N. C. Luhmann, and G. S. Nusinovich, Wiley, New York, 2005, Chap. 10.

[3] K. Jensen et al. "Emittance space charge and sharp electron sources," IVEC 2010.

[4] J. Yater et al. "Emission characterization of diamond current amplifier," IVEC 2010.

[5] J. Petillo et al. "Modeling emission processes in the finite-element MICHELLE gun \& collector simulation code," ibid.

[6] J. Pasour et al. "Sheet beam stick for low-voltage Wband EIK," ibid.

[7] C. Joye et al. "Microfabrication of a $220 \mathrm{GHz}$ grating for sheet beam amplifiers," ibid.

[8] P. Larsen et al. "Experimental characterization of a Kaband sheet-beam coupled-cavity slow-wave Structure," ibid.

[9] Y. Pchelnikov et al. "A novel millimeter-wave slowwave structure for longitudinal interaction with a sheet electron beam," ibid.

[10] K. Nguyen et al. "Design of a high-gain wideband high-power 220-GHz multiple-beam serpentine TWT," ibid.

[11] S. Cooke et al. "Nonlinear characterization of transverse interaction in sheet beam amplifiers," ibid.

[12] G. Stantchev et al. "Parallel simulation of complex waveforms in traveling-wave tube amplifiers," ibid.

[13] I. Chernyavskiy et al. "TESLA modeling of aftercavity interaction in high-efficiency klystrons," ibid.

[14] A. Vlasov et al. "2D modeling of beam-wave interaction in coupled cavity TWT with TESLA," ibid.

[15] S. Cooke et al. "A new complex envelope ADIFDTD algorithm for 3D simulations of slow wave structures," ibid.

[16] S. Ovtchinnikov et al. "High accuracy electron beam model development: MICHELLE eBEAM," ibid.

[17] D. Chernin et al. "A large signal model of extended interaction klystrons," ibid. 